\title{
Heritability of tibia fluctuating asymmetry and developmental instability in the winter moth (Operophtera brumata L.) (Lepidoptera, Geometridae)
}

\author{
STEFAN VAN DONGEN*†t, ELLEN SPRENGERS $\neq$, CHRISTER \\ LÖFSTEDT $\$ \&$ ERIK MATTHYSEN $\dagger$ \\ $\dagger$ Department of Biology, University of Antwerp (UIA), Universiteitsplein 1, B-2610 Wilrijk, Belgium, \\ $\ddagger$ Department of Ecology, Lund University, S-223 62 Lund, Sweden
}

\begin{abstract}
Broad-sense heritability of fluctuating asymmetry and developmental instability in the winter moth were analysed in a full-sib breeding experiment. Effects of both genetic background and common environment on both tibia FA (measured for the three pairs of legs) and body size were studied. As body size has previously been shown to be a reliable indicator of larval feeding success and expected fitness, the relationship between FA and body size was investigated as well. This relationship is of interest because it has been argued that the low heritability of FA results from a strong relationship between FA and fitness. Broad-sense $h^{2}$ of body size equalled zero whereas the effect of common environment was strong. The heritability of FA was low and not statistically significant for separate tibias. For FA based on the average of the three tibias $h^{2}$ equalled 0.07 and differed significantly from zero. The heritability of developmental instability equalled 0.09 . Thus the use of the hypothetical repeatability to translate the $h^{2}$ of FA to $h^{2}$ of developmental instability did not result in a strong increase in this species. Individual asymmetry was not correlated with fitness (as estimated by body size), indicating that the low heritabilities of FA are not a consequence of a strong correlation with fitness. Between-trait correlations in the unsigned FA were significant. However, these correlations are not necessarily indicative of an individual asymmetry parameter as the signed FA values were positively correlated as well, suggesting interdependent development of the three pairs of legs. Further research is necessary to investigate what the effects of interdependent development are on patterns in FA.
\end{abstract}

Keywords: developmental stability, fitness, fluctuating asymmetry, heritability, Operophtera, repeatability.

\section{Introduction}

Fluctuating asymmetry (FA, i.e. small random deviations from perfect bilateral symmetry) is often used as a measure of developmental instability. An individual's asymmetry has been argued to reflect or signal its fitness, health or heterozygosity. The extent to which asymmetry reflects genetic characteristics is of importance for evolutionary models of developmental instability. Therefore, the heritability of FA and of developmental instability are of interest for understanding and model-

*Correspondence: Fax: + 32382022 71;

E-mail: svdongen@uia.ua.ac.be ling their evolution. Recent surveys have revealed that although on average FA seems to have an additive genetic component, several studies fail to detect any heritability (Møller \& Thornhill, 1997). Møller \& Thornhill, (1997) argue by means of a meta-analysis that there is a small $(0.19)$ but highly significant additive component. Yet, several researchers have challenged this result and some have concluded that the heritability of FA is smaller (between 0 and 0.1, Leamy, 1997; Whitlock \& Fowler, 1997) or even zero (Markow \& Clarke, 1997). Several other studies, not included in the Møller \& Thornhill (1997) analysis, point in the same direction (Price et al., 1991; Fowler \& Whitlock, 1994; Brakefield \& Breuker, 1996; Windig, 1998; but see Blouw \& Boyd, 1992). Accurate estimation of heritabili- 
ties, for FA in particular, is difficult, and there is a lack of carefully controlled studies estimating heritability of FA (Falconer \& Mackay, 1996; Markow \& Clarke, 1997; Whitlock \& Fowler, 1997).

As FA is often correlated with fitness (Møller, 1997; but see Leung \& Forbes, 1996 and Clarke, 1998), a low heritability is expected (Møller \& Thornhill, 1997). Furthermore, if FA is to be used as a suitable estimate of stress the heritability of developmental instability must be low. On the other hand, the low heritability of FA may also be because FA is only poorly correlated with the underlying developmental instability and that low levels of heritability in FA are not necessarily indicative of low heritability in the underlying process (Whitlock, 1996). Large sample sizes, accurate measurement and within-subject repeats are required to obtain reliable estimates of both FA and its heritability. It is possible to translate patterns observed in FA to patterns in developmental instability with a bias correction using the hypothetical repeatability of FA (Whitlock, 1996; see Van Dongen, 1998 for a correction of the computational formula). This parameter $(R)$ estimates the betweenindividual variation in FA attributable to variation in developmental instability, relative to the total amount of variation in FA.

The analysis of the heritability of developmental instability via FA should follow two important steps. First, one should determine how much variation in FA results from between-individual variability (i.e. estimation of $R$ ) and, secondly, one should estimate to what extent this between-individual variation has a genetical basis. Only in that way can heritability estimates of developmental instability be obtained.

The population structure of the winter moth is to a large extent determined by the synchrony between egg hatching and budburst of its primary host (Quercus robur L.) (Varley et al., 1973; Van Dongen et al., 1997). In order to maximize fitness, caterpillar feeding must be initiated in synchrony with budburst, because leaf quality deteriorates rapidly in time resulting in lower feeding success. Asynchrony results in high larval mortalities, and also lower pupal and adult weights which in turn lead to higher pupal mortalities and both lower female egg content and shorter male lifespan, respectively (Varley et al., 1973; Gradwell, 1974; Van Dongen, 1997; Van Dongen et al., 1997; Van Dongen, unpubl. obs.). Thus, body size is determined largely by environmental conditions (i.e. feeding success) and is a reliable measure of fitness. Therefore, body size is expected to have a low heritability. In a recent study Van Dongen, (1997) did not find a negative relationship between male FA and body size, indicating that body size rather than FA is a reliable estimate of larval feeding success and expected fitness in this system. Such a negative relationship between FA and body size is, however, expected only if both developmental instability and body size have low heritability. Thus, in order to validate the conclusions found by Van Dongen, (1997) that FA is not a reliable estimator of fitness in the populations studied, the heritability of both FA and body size should be determined. In this paper we present results from a full-sib breeding experiment, estimating the heritability of both FA for three traits and body size. The relationship between FA and body size, as well as the between-trait correlation in FA are studied. Patterns in FA are translated to patterns in developmental instability using the hypothetical repeatability.

\section{Materials and methods}

\section{The winter moth}

The winter moth is a univoltine geometrid moth whose adults are active shortly after dusk in the late autumn (November in Belgium). The brachypterous females climb the nearest host tree (Quercus robur L.) where they copulate with the winged males. After copulation the female continues climbing into the canopy where the eggs are laid. Eggs hatch in spring and the caterpillars feed for 4-6 weeks before dropping to the ground on a silk thread for pupation in the soil (Varley et al., 1973).

\section{Moth rearing}

In November 1996, 32 copulating pairs were collected at night by hand on the lower parts of the trunk, in different oak woods in the vicinity of Antwerp, northern Belgium. The next day, males were frozen at $-80^{\circ} \mathrm{C}$ and females were allowed to lay eggs on a paper roll over the next 5-6 days. Females were frozen at $-80^{\circ} \mathrm{C}$ and eggs were stored outdoors. The next spring, hatching caterpillars were fed with young oak leaves and larvae from each clutch were randomly separated over two plastic trays $(1 \mathrm{~L})$ to allow estimation of common environment effects. Oak leaves were replaced every $2-3$ days and pupating individuals were stored in Eppendorf tubes and covered with $1 \mathrm{~cm}$ of soil to prevent dehydration. In October individuals were transferred to larger glass jars, and in November, emerging adults were stored at $-80^{\circ} \mathrm{C}$ before measurement of tibia asymmetry and body size (see below). Individuals from the different clutches were considered to be full-sibs. A count of spermatophores in females (as an estimate of number of copulations) collected in copula on the lower parts of the trunk confirmed that in these populations repeated mating is very infrequent (2, Van Dongen, unpubl. obs.). 


\section{Measurement of body size and asymmetry}

Asymmetry was measured for the tibias of the three pairs of legs (1: proto-; 2: meso-; 3: metatibia) of both males and females in parents and offspring. Before measurement legs were removed and squeezed between two glass microscope slides. Tibia length was measured under a microscope (enlargement $\times 30$ ) with a measureocular to the nearest $0.033 \mathrm{~mm}$ by one of us (E.S.). Two within-subject repeats on both sides were obtained to allow mixed-model analysis and separation of real asymmetry from measurement error (ME) (Palmer \& Strobeck, 1986), as well as to model and test heterogeneity in FA and ME (Van Dongen et al., 1999). Tibias were not remounted between successive measurements to avoid damage. We thus implicitly assume that the mounting process itself did not introduce much ME. Nevertheless it was necessary to press the tibias between the glass slides to avoid large amounts of ME as a result of bending of legs during storage in Eppendorf tubes. All legs of each individual moth were mounted simultaneously and measured in the same order. In this way there were always five measurements between two repeats. Body size was expressed as wing length for males and as body length for females which were measured to the nearest $0.1 \mathrm{~mm}$.

Analyses of FA were carried out following the guidelines in Palmer \& Strobeck, (1986) and Van Dongen et al., (1999). We used a mixed regression model approach to obtain estimates of the variance of signed FA and of ME (Van Dongen et al., 1999). The hypothetical repeatability of FA was obtained as $R=\left[1-\left(\left(V_{\mathrm{fa}} \times(\pi-2) / \pi\right) / V_{|\mathrm{fa}|}\right)\right]$, where $V_{\mathrm{fa}}$ and $V_{|\mathrm{fa}|}$ represent the variances of the signed and unsigned $F A$, respectively (Van Dongen, 1998).

Heritability of FA and of size was estimated by a fullsib analysis (Falconer \& Mackay, 1996) applying a nested random effects ANOVA (where the common environment was nested within family) and parentoffspring regressions (weighted for family size). The heritability as obtained from the ANOvA model is confounded with dominance variation and maternal effects and thus should be considered as an upper bound only (Falconer \& Mackay, 1996). Heritabilities were obtained for the three FA measures (FA1, FA2 and FA3 corresponding to the three tibias), the average FA and for body size. The latter was expressed by wing length and body length for males and females, respectively, by the average tibia lengths, and the average of these two measures. All statistical analyses were performed in SAS (version 6.12).

Unsigned individual FA values (i.e. estimates of individual developmental instability) are typically halfnormally distributed. This affects statistical testing of the variance components as these tests assume normality. We applied Box-Cox power transformations to obtain approximate normality.

Heritabilities and between-trait correlations of the unsigned FA were translated to patterns in the presumed underlying developmental instability following Whitlock, (1996, 1998).

\section{Results}

\section{Body size}

Variability in body size is summarised in Fig. 1. For both males and females, body size was smaller in the offspring. Female tibias were shorter than male tibias (ANOVA: both $P<0.0001$ ). This difference between males and females was the same in parents and offspring (two-way interaction: $P=0.9$ ).

Table 1 summarizes the variance components for the nested random effects ANOVA with family and common environment (nested within family) as random factors. Body size was standardized for both sexes prior to the analysis to partial out differences between males and females. Heritabilities were zero (even $<0$ ) in all cases whereas the common environment effect was highly significant and explained between 35 and 50 of the total variation. The family means were not significantly correlated with the mid-parent body sizes for any of the three size measures (all $P>0.2$ ).

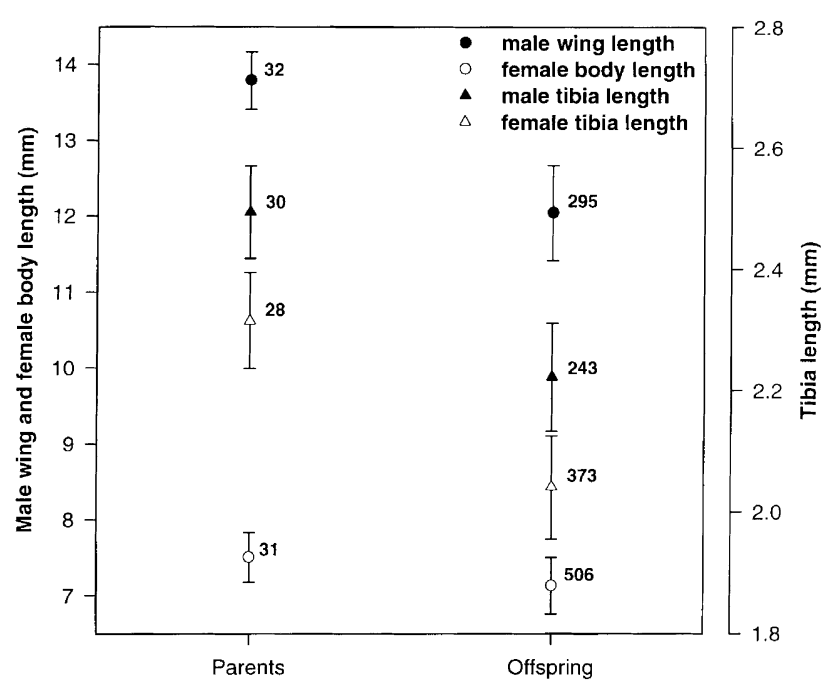

Fig. 1 Variation in size of male and female winter moths (wing and body length and average tibia length) for parents and offspring. Error bars represent standard deviation divided by 2. Sample sizes are indicated next to the symbols. 
Table 1 Variance components of a nested ANova model of the two size measures (average tibia length and wing/body length) of winter moths and their average. Significance tests of the variance components were performed by likelihood ratio tests $\left({ }^{* * *} P<0.0001\right)$. Sizes were standardized per sex. The Shapiro-Wilks statistic $(W)$ indicated approximate normality. The causal components corresponding to the different variances are indicated within brackets. [ $V_{\mathrm{A}}$, additive variance; $V_{\mathrm{D}}$, dominance variance; $V_{\mathrm{ECl}}$, variance attributable to common environment (i.e. common tray in this study); $V_{\mathrm{EC} 2}$, all other sources of common environment (e.g. maternal effects); $V_{\mathrm{EW}}$, residual variance]

\begin{tabular}{lccc}
\hline & Tibia & Wing/body & Average size \\
\hline Family $\left(1 / 2 V_{\mathrm{A}}+1 / 4 V_{\mathrm{D}}+V_{\mathrm{EC} 2}\right)$ & -0.06 & -0.10 & -0.11 \\
Common environment $\left(V_{\mathrm{EC} 1}\right)$ & $0.37^{* * *}$ & $0.57^{* * *}$ & $0.46^{* * *}$ \\
Residual $\left(V_{\mathrm{EW}}\right)$ & 0.68 & 0.56 & 0.48 \\
$W$ & 0.95 & 0.99 & 0.96 \\
Sample size & 672 & 516 & 509 \\
\hline
\end{tabular}

\section{Fluctuating asymmetry}

Distribution, significance and repeatability of FA Table 2 summarizes the variance components describing size variation as obtained from mixed regression models. For all three tibias and in both parents and offspring ME was much lower relative to FA. Consequently, FA was highly significant. There appeared to be differences in FA between the two sexes. In one case (tibia 1 of the offspring) males were more asymmetrical than females, whereas in all other cases females were (or tended to be) more asymmetrical. Also note that asymmetry was an order of magnitude larger in the offspring, compared to the parents, a difference that was highly significant in all cases (likelihood ratio test: $\mathrm{P}<0.01$ ). After sequential Bonferroni correction for repeated testing (Hochberg, 1988) none of the directional asymmetry tests was significant. Measurement error differed little between the sexes. Because testing heterogeneity in ME was based on all observations rather than on the number of individuals, this test has very high power so that very small differences can be detected. Because ME was small relative to FA and because the magnitude of heterogeneity was very small, we used the traditional unsigned

Table 2 Overview of mixed regression model analysis of asymmetry in winter moth parents and offspring for the three tibias (FA1, FA2, FA3). Estimates of size variation between individuals $\left(V_{\text {ind }}\right)$, of fluctuating asymmetry $\left(V_{\mathrm{fa}}\right)$ and of measurement error $\left(V_{\mathrm{me}}\right)$ were obtained. The significance of $V_{\mathrm{fa}}\left({ }^{* * *} P<0.001\right)$ and the significance of heterogeneity in FA and ME between males and females were tested with the likelihood ratio test. Directional asymmetry (DA) was tested with an $F$-test. Sample sizes $(N)$ indicate the number of individuals incorporated in the analysis

\begin{tabular}{|c|c|c|c|c|c|c|}
\hline & Sex & $V_{\text {ind }}$ & $V_{\mathrm{fa}}$ & $V_{\mathrm{me}}$ & DA ( $P$-value $)$ & $N$ \\
\hline \multicolumn{7}{|l|}{ Parents } \\
\hline \multirow[t]{3}{*}{ FA1 } & $\mathrm{m}$ & 2.2 & $0.041 * * *$ & 0.006 & 0.2 & 31 \\
\hline & $\mathrm{f}$ & 2.6 & $0.063 * * *$ & 0.006 & 0.8 & 31 \\
\hline & Heterogeneity test: & & $P=0.09$ & $P>0.5$ & & \\
\hline \multirow[t]{3}{*}{ FA2 } & $\mathrm{m}$ & 2.8 & $0.027 * * *$ & 0.006 & 0.2 & 31 \\
\hline & $\mathrm{f}$ & 2.5 & $0.046^{* * *}$ & 0.005 & 0.1 & 31 \\
\hline & Heterogeneity test: & & $P=0.10$ & $P>0.5$ & & \\
\hline \multirow[t]{3}{*}{ FA3 } & $\mathrm{m}$ & 2.0 & $0.045 * * *$ & 0.006 & 0.004 & 31 \\
\hline & $\mathrm{f}$ & 2.6 & $0.190 * * *$ & 0.005 & 0.7 & 31 \\
\hline & Heterogeneity test: & & $P<0.01$ & $P>0.5$ & & \\
\hline \multicolumn{7}{|c|}{ Offspring } \\
\hline \multirow[t]{3}{*}{ FA1 } & $\mathrm{m}$ & 3.7 & $1.44 * * *$ & 0.01 & 0.05 & 276 \\
\hline & $\mathrm{f}$ & 3.2 & $0.68 * * *$ & 0.05 & 0.8 & 450 \\
\hline & Heterogeneity test: & & $P<0.001$ & $P<0.01$ & & \\
\hline \multirow[t]{3}{*}{ FA2 } & $\mathrm{m}$ & 4.0 & $0.58 * * *$ & 0.01 & 0.8 & 280 \\
\hline & $\mathrm{f}$ & 3.1 & $0.93 * * *$ & 0.02 & 0.4 & 440 \\
\hline & Heterogeneity test: & & $P<0.001$ & $P>0.1$ & & \\
\hline \multirow{3}{*}{ FA3 } & $\mathrm{m}$ & 2.5 & $0.38 * * *$ & 0.04 & 0.6 & 278 \\
\hline & $\mathrm{f}$ & 2.9 & $0.46 * * *$ & 0.06 & 0.9 & 428 \\
\hline & Heterogeneity test: & & $P<0.001$ & $P<0.01$ & & \\
\hline
\end{tabular}


FA (absolute value of left minus right) as estimate of individual FA.

Descriptive statistics of the distribution of the signed (left minus right) and unsigned FA are given in Table 3. For parents, the signed FA was approximately normally distributed and the repeatability was consequently low. For the offspring, however, the signed FA was leptokurtically distributed, especially for tibias 1 and 2, and repeatability was much higher than for the parents. This indicates that the between-individual component of FA was more important in the offspring. This betweenindividual component was not entirely attributable to the differences between males and females as within the sexes the distribution of the signed FA was still leptokurtic with a relatively high repeatability.

FA (for the three tibias and the average) was not correlated with individual size (tibia length, wing/body length, average) for parents and offspring separately. After sequential Bonferroni correction none of the 24 correlations was significant. Correlations were performed correcting for sex differences in FA and size. Analyses per sex gave similar results (not shown). Therefore, no size scaling was introduced. Pooling all data resulted in significant negative correlations for males (eight out of 12 significant after sequential Bonferroni) but not for females (none out of 12 significant after sequential
Bonferroni) (Fig. 2). There were small but significant correlations between the signed FA of different tibias for the offspring $(1-2: \quad r=0.19, \quad P<0.0001 ; \quad 1-3$ : $r=0.12, \quad P=0.002 ; 2-3: \quad r=0.14, \quad P=0.0005$ ), but not for the parents. Yet, the correlation coefficients did not differ between parents and offspring (all $P$ 0.4) possibly because of the lower sample sizes for the parents. Correlations between unsigned FA of the different tibias were as follows: $1-2: \quad r=0.22$, $P<0.0001 ; 1-3: \quad r=0.12, \quad P=0.002 ; 2-3: r=$ $0.06, P=0.15$. These correlations were calculated partialling out differences between males and females. Translating these correlations to patterns in presumed underlying developmental instability using the average repeatabilities over the two sexes resulted in: 1-2: $r=0.46,1-3: r=0.37,2-3: r=0.18$. For the parents none of the correlations between the unsigned FAs was significant. This could have been expected because both the repeatabilities and the sample sizes for the parents were much lower.

Heritability of FA Heritability estimates of FA as obtained from two-way nested random ANOVA models are given in Table 4. For the untransformed unsigned FA only one was significant (at the 0.05 level, thus not after sequential Bonferroni). As the data were nonnormal (Table 4) we used Box-Cox power transforma-

Table 3 Distribution of signed and unsigned asymmetry for winter moth parents and offspring for the three tibias (FA1, FA2, FA3). Descriptive statistics were obtained for the complete samples and for males and females separately. Different columns represent: the variance (confounded with measurement error) $\left(V_{\mathrm{fa}}+\mathrm{me}\right)$, Shapiro-Wilks statistic $(W)$, kurtosis $(K)$, and skewness $(S)$ for the signed FA; the coefficient of variation $(C V)$, and the variance $\left(V_{|\mathrm{fa}|}\right)$ of the unsigned FA; and the hypothetical repeatability $(R)$ of individual asymmetry

\begin{tabular}{ccccccccc}
\hline & & \multicolumn{2}{c}{ Signed FA } & & & Unsigned FA & \\
& Sex & $V_{\mathrm{fa}+\mathrm{me}}$ & $W$ & $K$ & $S$ & $C V$ & $V_{\text {|fa }}$ & $R$ \\
\hline Parents & & & & & & & & \\
FA1 & $\mathrm{m}$ & 0.047 & 0.97 & -0.7 & -0.2 & 0.79 & 0.018 & 0.07 \\
& $\mathrm{f}$ & 0.069 & 0.98 & -0.2 & -0.3 & 0.79 & 0.026 & 0.04 \\
& All & 0.058 & 0.98 & -0.4 & -0.2 & 0.79 & 0.022 & 0.05 \\
FA2 & $\mathrm{m}$ & 0.033 & 0.97 & 0.1 & -0.4 & 0.89 & 0.015 & 0.20 \\
& $\mathrm{f}$ & 0.051 & 0.98 & 0 & 0.3 & 0.73 & 0.021 & 0.12 \\
& All & 0.042 & 0.98 & -0.2 & -0.1 & 0.81 & 0.019 & 0.19 \\
FA3 & $\mathrm{m}$ & 0.051 & 0.99 & 0.3 & 0.2 & 0.78 & 0.025 & 0.26 \\
& $\mathrm{f}$ & 0.197 & 0.96 & -0.5 & 0.2 & 0.69 & 0.062 & $<0$ \\
& All & 0.120 & 0.96 & 0.6 & 0.6 & 0.79 & 0.049 & 0.12 \\
Offspring & & & & & & & \\
FA1 & $\mathrm{m}$ & 1.40 & 0.72 & 29.9 & -2.7 & 1.73 & 1.06 & 0.52 \\
& $\mathrm{f}$ & 0.68 & 0.88 & 10.5 & 0.3 & 1.30 & 0.43 & 0.42 \\
FA2 & All & 0.95 & 0.79 & 28.5 & -1.8 & 1.53 & 0.67 & 0.48 \\
& $\mathrm{~m}$ & 0.59 & 0.77 & 40.8 & 3.8 & 1.49 & 0.40 & 0.47 \\
& $\mathrm{f}$ & 0.86 & 0.77 & 20.0 & -2.0 & 1.54 & 0.61 & 0.49 \\
FA3 & All & 0.75 & 0.78 & 25.6 & -0.4 & 1.54 & 0.53 & 0.48 \\
& $\mathrm{~m}$ & 0.42 & 0.95 & 4.7 & -0.6 & 1.02 & 0.21 & 0.28 \\
& $\mathrm{f}$ & 0.51 & 0.99 & 1.3 & 0.0 & 0.86 & 0.22 & 0.15 \\
& All & 0.48 & 0.97 & 2.3 & -0.2 & 0.93 & 0.22 & 0.21 \\
\hline
\end{tabular}




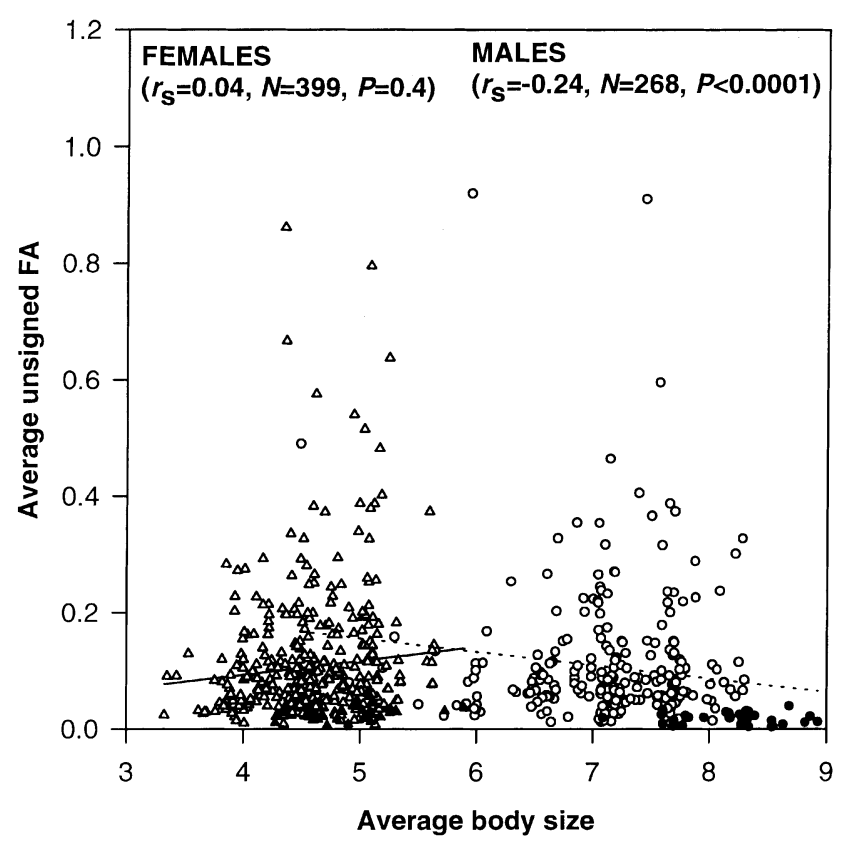

Fig. 2 Relationship between average body size and average unsigned FA for male (circles) and female (triangles) winter moths. Offspring are represented by open and parents by black symbols. Regression lines show the relationship between FA and body size for males (dotted line) and females (solid line). Note that the difference in body size for males and females is to a large extent attributable to the fact that different measures were taken for the two sexes (see text and Fig. 1), but also to real size differences in male and female tibia lengths (Fig. 1).

tions to normalize them. This appeared to work well as judged from the Shapiro-Wilks statistics which were higher than 0.95 for the transformed data, indicating approximate normality (Table 4). This transformation yielded very similar conclusions, except that the heritability for the average FA became significant even after correction for multiple testing. In both analyses, the common environment effect was never significant and often even negative (Table 4). Translating the observed heritabilities for one tibia (estimates from the transformed data) to one that would be expected if based on the average FA from three traits, using the formula $h_{\mathrm{m}}^{2}=h_{1}^{2} \times(m /(1+(m-1) \times R)) \quad(m=$ number of traits, Whitlock, 1996), we obtained values close to the observed heritability of this average FA (Table 4). Translating the observed heritabilities of FA to the expected one for developmental instability (i.e. $m=\infty$ ) resulted in a heritability of 0.09 (average of three estimates corresponding to the three tibias, Table 4).

Parent-offspring regressions of the unsigned FA values were not significant after sequential Bonferroni. Ten slopes were positive and six were negative. The frequency of positive ones is not significantly larger than expected by chance (binomial test, $P=0.12$ ).

\section{Discussion}

\section{Heritability of fluctuating asymmetry and developmental instability}

This study has shown that the heritability of a singletrait fluctuating asymmetry $\left(h^{2}=0.03\right.$, but not significant) is of the order of magnitude found in other carefully controlled studies (Brakefield \& Breuker, 1996; Leamy, 1997; Markow \& Clarke, 1997; Whitlock \& Fowler, 1997; Windig, 1998). Heritability based on the average FA of the three tibias equalled 0.07 and was statistically significant. Translating these heritabilities of FA to one for developmental instability resulted in a heritability of 0.09 , only a slight increase. The typically low heritability found here therefore cannot be attributed to a low repeatability of FA.

Breeding conditions appeared to be suboptimal, as judged from the lower body size of the offspring compared to the parents, and resulted in a higher degree of both FA and the between-individual differences in the underlying developmental instability (as judged from the relatively higher repeatabilities in the offspring). Thus, although individuals appeared to differ in their ability to stabilize their development under the breeding conditions, this did not appear to have a strong genetical basis.

The hypothetical repeatability can be used to translate patterns in FA to patterns in developmental instability after making the assumption that the development of each trait is independent (Whitlock, 1996). This assumption may be violated here. We found significant positive correlations between the signed FAs of the different tibias, which may indicate that the development of these different traits is not independent. The level and direction of asymmetry of one tibia appears to depend on the development of the other tibias. This adds an extra source of variability to the observed FA values which may have resulted in an incorrect estimation of the hypothetical repeatability so that an erroneous bias correction was carried out. To what extent this problem is general and how it affects the repeatability of FA should become a topic of further research.

\section{Correlation between FA and fitness}

Body size is a reliable estimate of larval feeding success and the expected fitness of an individual. The smaller body size of the offspring compared to the parents indicates that the rearing conditions were suboptimal. This appeared to result in an increase of FA for the three tibias at the population level. The presence of such a pattern suggests the presence of a so-called population asymmetry parameter (e.g. Leamy, 1993). Yet, in spite of 
Table 4 Heritability estimates of fluctuating asymmetry of the three tibias of winter moths (FA1, FA2, FA3) and the average value $($ avFA) based on the variance components $(\times 100)$ of a nested ANOvA model. Causal components corresponding to the different variances are indicated within brackets (details in Table 1). Variance components were tested for significance by the likelihood ratio test. In all analyses, gender was added as a covariate to control for differences between the two sexes.

$W$ represents the Shapiro-Wilks statistic. Values lower than 0.95 were used as an indication of non-normality. Box-Cox power transformations were applied in that case. Significant heritabilities are indicated in bold. Observed heritabilities of FA were transformed to their expected values if three $\left(h_{3}^{2}\right)$ and an infinite $\left(h_{\mathrm{inf}}^{2}\right)$ number of traits had been measured

\begin{tabular}{|c|c|c|c|c|}
\hline & FA1 & FA2 & FA3 & avFA \\
\hline \multicolumn{5}{|l|}{ Untransformed data } \\
\hline Family $\left(1 / 2 V_{\mathrm{A}}+1 / 4 V_{\mathrm{D}}+V_{\mathrm{EC} 2}\right)$ & 0.01 & 0.006 & -0.001 & 0.006 \\
\hline Common environment $\left(V_{\mathrm{EC} 1}\right)$ & -0.04 & -0.01 & 0.03 & -0.01 \\
\hline Residual ( $\left.V_{\mathrm{EW}}\right)$ & 0.78 & 0.54 & 0.22 & 0.21 \\
\hline$h^{2}$ & 0.01 & 0.01 & 0 & 0.05 \\
\hline$P$-value & 0.2 & 0.2 & 0.4 & 0.05 \\
\hline$W$ & 0.58 & 0.56 & 0.85 & 0.76 \\
\hline \multicolumn{5}{|l|}{ Transformed data } \\
\hline Family $\left(1 / 2 V_{\mathrm{A}}+1 / 4 V_{\mathrm{D}}+V_{\mathrm{EC} 2}\right)$ & 0.08 & 0.42 & 0.10 & 0.80 \\
\hline Common environment $\left(V_{\mathrm{EC} 1}\right)$ & -0.7 & -0.2 & 0.05 & -0.11 \\
\hline Residual ( $\left.V_{\text {EW }}\right)$ & 22.6 & 18.9 & 7.4 & 21.6 \\
\hline$h^{2}$ & 0.01 & 0.04 & 0.03 & 0.07 \\
\hline$P$-value & 0.4 & 0.1 & 0.1 & 0.01 \\
\hline Box-Cox lambda & 0.3 & 0.3 & 0.4 & 0.15 \\
\hline$W$ & 0.97 & 0.97 & 0.98 & 0.99 \\
\hline$h_{3}^{2}$ & 0.02 & 0.06 & 0.06 & - \\
\hline$h_{\mathrm{inf}}^{2}$ & 0.03 & 0.08 & 0.14 & - \\
\hline Sample size & 607 & 606 & 592 & 585 \\
\hline
\end{tabular}

the very high sample sizes and the large between-individual variation in both body size (strong effect of breeding and of common environment) and FA [high repeatabilities (but see above)], we find a correlation between FA and body size at the individual level only in males and when parents and offspring are pooled. A closer examination of Fig. 2, however, reveals that the difference in FA between parents and offspring cannot be explained by size differences alone. The FA of the offspring is much higher compared to parents of the same size, indicating that other factors, besides food quality (which is thought primarily to affect body size), during the rearing may have increased FA in the offspring. Therefore, the significant correlations between size and FA in males for the pooled dataset may not be very meaningful here.

Earlier studies have shown that body size is a reliable estimate of fitness. Pupal mortality decreases with pupal weight (see also Gradwell, 1974), larger females produce more eggs (Van Dongen, 1997), larger adults produce clutches that are better locally adapted to the phenology of their individual hosts (Van Dongen, 1997; Van Dongen et al., 1997), male and female lifespan increases with body size and larger individuals have a slight mating advantage (Van Dongen, unpubl. obs.). If individual asymmetry reflects fitness, a correlation with body size is expected. However, both at the common environment and the individual level such a relationship is lacking, suggesting that individual asymmetry does not reflect fitness in the winter moth. This was also confirmed by directly relating individual asymmetry with several fitness components (Van Dongen, unpubl. obs.). Thus, the low $h^{2}$ of FA and of developmental instability cannot be attributed to a strong relationship between FA and fitness.

The positive association between the unsigned FA of the three tibias suggests the presence of an individual asymmetry parameter (i.e. IAP, e.g. Leamy 1993). However, these correlations are confounded with the correlations between the signed FA values, which makes the interpretation of the patterns difficult and argues against the presence of an IAP. Recent reviews have indicated that an IAP is more frequently encountered when closely related characters are studied (Leamy, 1993). Such traits may have an interdependent development as seems to be the case here. The higher frequency of an IAP in related traits could indicate that some of these correlations between the unsigned FAs are in fact the result of an association between the signed FAs. 


\section{Conclusions}

In conclusion, this study provides evidence for a low heritability of FA. The magnitude of $h^{2}$ was comparable to that found in many other studies. The heritability of developmental instability was low as well. The low $h^{2}$ values found in this study cannot be attributed to an association between individual asymmetry and fitness. The application of $R$ to translate heritability of FA into heritability of developmental instability is somewhat troublesome considering the positive associations between the signed FAs for the three tibias which indicate interdependent development of the three tibias. Further research should focus on the importance and effects of interdependent development on the hypothetical repeatability of FA.

\section{Acknowledgements}

S.V.D. is research assistant and E.M. research associate with the Science Fund Flanders (FWO vlaanderen). This study was partially funded by the Swedish National Science Research Council and the Knut and Alice Wallenberg foundation. We thank Jack Windig, Luc Lens, Thierry Backeljau and Robby Stoks for their comments on earlier drafts of the manuscript.

\section{References}

BLOUW, D. M. AND BOYD, G. J. 1992. Inheritance of reduction, loss, and asymmetry of the pelvis in Pungitius pungitius (ninespine stickleback). Heredity, 68, 33-42.

BRAKEFIELD, P. M. AND BREUKER, C. J. 1996. The genetical basis of fluctuating asymmetry for developmentally integrated traits in a butterfly eyespot pattern. Proc. R. Soc. B, 263, 1557-1563.

ClARKe, G. M. 1998. Developmental stability and fitness: the evidence is not quite so clear. Am. Nat., 152, 762-766.

FALCONER, D. S. AND MACKAY, T. F. C. 1996. Introduction to Quantitative Genetics. Longman, Essex.

FOWLER, K. AND WHITLOCK, M. J. 1994. Fluctuating asymmetry does not increase with moderate inbreeding in Drosophila melanogaster. Heredity, 73, 373-376.

GRADWELL, G. R. 1974. The effects of defoliators on tree growth. In: Morris, M. G. and Perring, F. H. (eds) The British Oak. Its History and Life History, pp. 182-193. F.W. Classey, Flaringdon.

HOCHBERG, Y. 1988. A sharper Bonferroni procedure for multiple tests of significance. Biometrika, 75, 800-802.
LEAMY, L. 1993. Morphological integration of fluctuating asymmetry in the mouse mandible. Genetica, 89, 139-153.

LEAMY, L. 1997. Is developmental stability heritable? J. Evol. Biol., 10, 21-29.

LEUNG, B. AND FORBES, M. R. 1996. Fluctuating asymmetry in relation to stress and fitness: effect of trait type as revealed by meta-analysis. Ecoscience, 3, 400-413.

MARKow, T. A. AND ClARKe, G. M. 1997. Meta-analysis of the heritability of developmental stability: a giant step backward. J. Evol. Biol., 10, 31-37.

MøLlER, A. P. 1997. Developmental stability and fitness: a review. Am. Nat., 149, 916-932.

MøLlER, A. P. AND THORNHILl, R. 1997. A meta-analysis of the heritability of developmental stability. J. Evol. Biol., 10, 1-16.

PALMER, A. R. AND STROBECK, C. 1986. Fluctuating asymmetry: measurement, analysis, patterns. Ann. Rev. Ecol. Syst., 17, $391-421$.

PRICE, T., CHI, E., PAVElKA, M. AND HACK, M. 1991. Population and developmental variation in the feather tip. Evolution, 45, $518-533$.

VAN DONGEN, s. 1997. The population structure of the winter moth (Operophtera brumata L.) in relation to local adaptation and habitat fragmentation. PhD Dissertation, University of Antwerp.

VAN DONGEN, S. 1998. How repeatable is the estimation of developmental stability by fluctuating asymmetry? Proc. $R$. Soc. B, 265, 1423-1427.

VAN DONGEN, S., BACKELJAU, T., MATTHYSEN, E. AND DHONDT, A. A. 1997. Synchronization of hatching date with budburst of individual host trees (Quercus robur) in the winter moth (Operophtera brumata) and its fitness consequences. J. Anim. Ecol., 66, 113-121.

VAN DONGEN, S., MOLENBERGHS, G. AND MATTHYSEN, E. 1999. Statistical analysis of fluctuating asymmetry: REML estimation of a mixed regression model. J. Evol. Biol., 12, 94102.

VARLEY, G. C., GRADWELl, G. R. AND HASSELL, M. P. 1973. Insect Population Ecology. Blackwell Scientific Publications, Oxford.

WHITLOCK, M. 1996. The heritability of fluctuating asymmetry and the genetic control of developmental stability. Proc. $R$. Soc. B, 263, 849-854.

WHITLOCK, M. 1998. The repeatability of fluctuating asymmetry: a revision and extension. Proc. R. Soc. B, 265, 14281430 .

WHITLOCK, M. AND FOWLER, K. 1997. The instability of studies of instability. J. Evol. Biol., 10, 63-67.

WINDIG, J. J. 1998. Evolutionary genetics of fluctuating asymmetry in the peacock butterfly (Inachis io). Heredity, 80, $382-392$. 